\title{
Enfermedad de Moyamoya, a propósito de dos casos
}

\author{
LETICIA YÁÑEZ P. ${ }^{1}$, MARCELA PAREDES M. ${ }^{2}$, MICHELANGELO LAPADULA A. ${ }^{1}$, \\ PATRICIO MARTÍNEZ T. ${ }^{3}$, FERNANDO DURÁN H. ${ }^{4}$ \\ 1. Médico UPCP, Clínica Santa María. \\ 2. Neuróloga Infantil, Clínica Santa María. \\ 3. Neurocirujano Jefe, Clínica Santa María. \\ 4. Neurorradiólogo, Clínica Santa María.
}

\begin{abstract}
Moyamoya disease, 2 case-report

Moyamoya Disease is a progressive occlusive cerebrovascular disease of both internal carotid arteries and their branches, with compensatory development of a fine collateral vascular network. The etiology of the disease is unknown; however, several studies have suggested involvement of genetic and environmental factors in its pathogenesis. In children, it causes transient ischemic attacks and cerebral infarction, while bleeding is more frequent in adults. In Japan, where the disease is most frequently observed, the development of magnetic resonance with angiography has allowed diagnosis in asymptomatic Moyamoya Disease. Revascularization surgery is believed to reduce the incidence of new ischemic events and improve long term prognosis in these patients. We present 2 children with Moyamoya Disease treated in our Clinic between 1998 and 2007.

(Key words: Moyamoya, ischemic attacks, cerebral infarction, vasculitis).

Rev Chil Pediatr 2008; 79 (6): 629-635
\end{abstract}

\section{RESUMEN}

La enfermedad de Moyamoya es una enfermedad cerebrovascular oclusiva, progresiva de ambas arterias carótidas internas o sus ramas, compensada por el desarrollo de una fina red vascular colateral. Su etiología es desconocida, aunque distintos estudios han asociado su patogénesis a factores genéticos y ambientales. En Pediatría se manifiesta generalmente como crisis isquémicas transitorias o infartos cerebrales, mientras que en adultos es más frecuente la hemorragia cerebral ${ }^{1,2}$. En países donde es más frecuente, como Japón, se ha logrado hacer un diagnóstico precoz en pacientes aún asintomáticos, gracias al desarrollo de la Resonancia magnética con angiografía cerebral². La cirugía de revascularización lograría reducir la incidencia de nuevos episodios isquémicos y mejorar el pronóstico a largo plazo de los pacientes que padecen esta patología ${ }^{3}$. Presentamos a continuación dos casos que han llegado a nuestro centro en un período de 9 años.

(Palabras clave: Moyamoya, isquemia cerebral, infarto cerebral, vasculitis).

Rev Chil Pediatr 2008; 79 (6): 629-635

Trabajo recibido el 12 de mayo de 2008, devuelto para corregir el 10 de julio de 2008, segunda versión el 10 de octubre de 2008, aceptado para publicación el 17 de noviembre de 2008.

Correspondencia a:

Leticia Yáñez Palma

E-mail: lyanez@csm.cl 


\section{Introducción}

La enfermedad de Moyamoya (EM) es una vasculopatía que se caracteriza por una estenosis progresiva, (hasta su oclusión), de la porción terminal de ambas carótidas internas, que puede incluir a arterias cerebrales anterior (ACA) y media (ACM), asociada a una red vascular anormal de vasos denominados de moyamoya. Se trata de una enfermedad poco frecuente en pediatría, por lo que el objetivo de esta publicación es ponerla en mente como diagnóstico diferencial en cuadros de sintomatología de origenvascular, orientando el estudio dirigidamente a su búsqueda. Lo anterior cobra mayor relevancia si se considera que es una patología progresiva, que diagnosticada precozmente, permite controlar su evolución y evitar deterioro clínico que puede llegar a ser invalidante, o incluso con consecuencias fatales.

Susuki y Takaku ${ }^{4}$, en 1969 nominaron a esta entidad clínica," humo de volcanes", en japonés, moyamoya, debido a la apariencia que tienen las imágenes angiográficas. Su etiología aún es desconocida, aunque estudios epidemiológicos sugieren factores genéticos en su patogénesis.

En niños generalmente se presenta como crisis isquémicas transitorias o accidentes vasculares, con progresión que puede ser lenta, con eventos intermitentes, o fulminante con rápido compromiso neurológico. El diagnóstico se realiza sobre la base de los hallazgos clínicos y radiológicos que evidencian la estenosis característica de arterias carótidas internas junto con abundantes vasos colaterales. La evolución sin tratamiento es inevitablemente a la progresión del compromiso vascular y clínico.

La cirugía de revascularización cerebral mejora su pronóstico a largo plazo, las distintas técnicas descritas tienen la finalidad de prevenir mayor daño isquémico aumentando el flujo sanguíneo colateral a través de la carótida externa.

Describimos a continuación nuestra experiencia con dos pacientes a quienes se hizo diagnóstico de EM en la Unidad de Paciente Crítico Pediátrico de Clínica Santa María (UPCP-CSM) entre Enero de 1998 y Diciembre de 2007. En este período han egresado 17 pacientes con Accidente Vascular Encefálico
(AVE), de un total de 5864 pacientes hospitalizados, correspondiendo la EM al 11\% de los AVE en pacientes entre 15 días y 17 años, con una incidencia de $0,2 \%$ por centro por año.

\section{Caso 1}

Escolar de sexo femenino, con antecedentes de cefalea vascular de 2 años de evolución, y un mes previo a su ingreso, parestesias y falta de fuerzas de pocas horas de duración con regresión completa. TAC de cerebro sin contraste normal. Consultó en CSM el 24 de Octubre de 2001, a la edad de 12 años 2 meses, por cuadro de cefalea de 4 días de evolución, holocránea, pulsátil, de intensidad progresiva. El día de su ingreso amaneció con falta de fuerzas en extremidad superior izquierda, haciéndose extensiva en pocas horas a todo el hemicuerpo. TAC de cerebro mostró hipodensidad frontal derecha.

Angioresonancia evidenció infarto corticosubcortical frontal derecho; oclusión de arteria ACM y oclusión casi total de carótida derecha (figura 1). Se planteó diagnóstico de Vasculitis, administrándose Gammaglobulina y Metilprednisolona. Se realizó angiografía convencional que mostró oclusión en bifurcación de carótida derecha y oclusión ACA izq, con colaterales desde cerebral posterior, sin colaterales de carótida externa, compatible con EM.

Permaneció hospitalizada hasta el 15 de noviembre, dándose de alta con hemiparesia izq mínima. La semana siguiente de su alta fue intervenida quirúrgicamente en Instituto de Neurocirugía, donde se realiza encefaloduroarterio anastomosis derecha, y en un segundo acto quirúrgico a izquierda. Ha evolucionado sin nuevos eventos de vasculares, con episodios de cefalea de carácter vascular con adecuado rendimiento académico, sin déficit motor. Se ha mantenido con Flunarizina, el control de angioresonancia se muestra en figura 2.

\section{Caso 2}

Preescolar de 4 años 3 meses con antecedentes de retraso del desarrollo psicomotor leve, de predominio en área del lenguaje expresivo. TAC de cerebro 2004 normal. Consultó en CSM el 3 de Junio de 2007, por presentar crisis tónico clónico heemigeneralizada izquierda de 
una hora de duración, quedando con hemiparesia facio braquio crural (FBC) del mismo lado. Se realizó EEG normal y estudio de trombofilia que resultaron normales. Angio- RNM mostró alteración de perfusión mayor hemisferio izquierdo. La angiografía convencional (figura 3 ) mostró estrechamiento arterial en bifurcación de ambas carótidas internas, aguzamiento de ambos sifones, severa estenosis de ACM y ACA derecha, comunicantes posteriores abiertas, extensa red colateral ganglionar, anastomosis corticortical frontal, infartos corticales antiguos bifrontales. Evolucionó con regresión de hemiparesia compatible con EM. Se realizó trepanación de hemicalota bilateral simétrica. Veintiún trepanaciones con apertura de duramadre e inversión. Evoluciona sin nuevos epi-

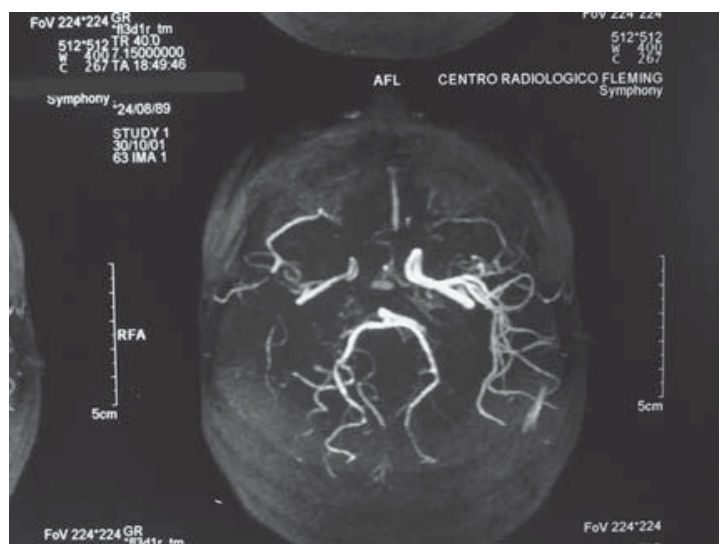

Figura 1. Angioresonancia preoperatoria de caso $\mathrm{n}^{\mathrm{o}} 1$.

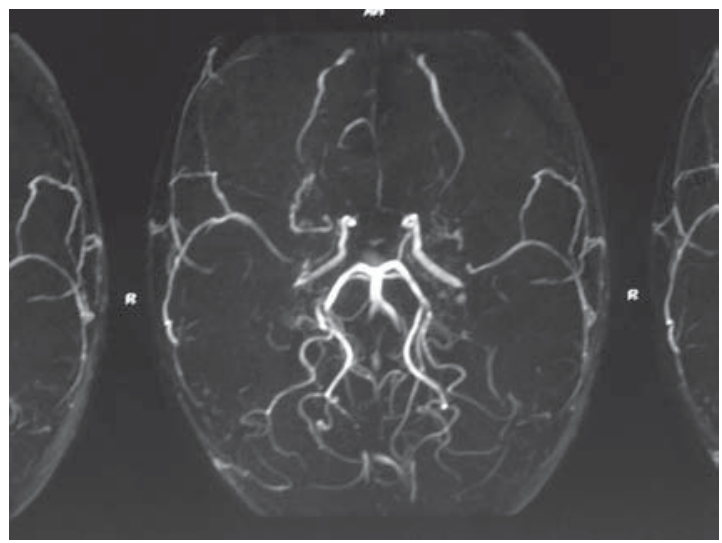

Figura 3. Angioresonancia preoperatoria de caso $\mathrm{n}^{\circ} 2$. sodios vasculares y con aceleración del desarrollo motor y del lenguaje, control de angioresonancia se muestra en figura 4.

\section{Epidemiología}

La EM fue descrita como tal por primera vez en Japón, lugar en donde es la primera causa de enfermedad cerebrovascular en la población infantil ${ }^{5}$, con una incidencia de $3 \%$ porcentro por año, a diferencia de la descrita en Europa que es diez veces menor ${ }^{6}$. Afecta dos veces más a las mujeres. Se describe una distribución etárea bimodal, un grupo en la primera década de la vida, y un segundo grupo en adultos entre los 30 y 40 años ${ }^{7}$.

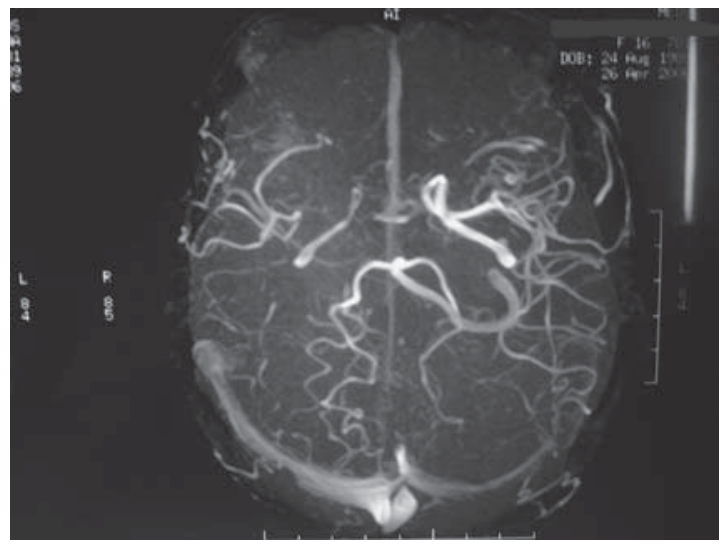

Figura 2. Angioresonancia postoperatoria de caso $\mathrm{n}^{\circ} 1$.

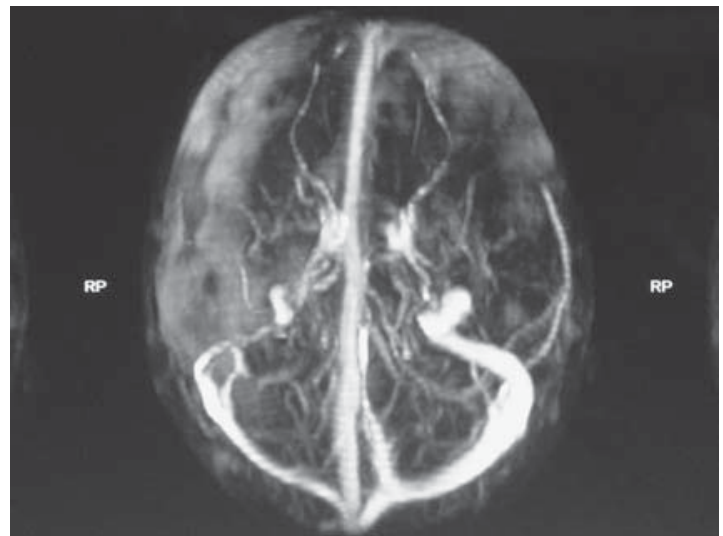

Figura 4. Angioresonancia postoperatoria de caso $\mathrm{n}^{\mathrm{o}} 2$. 


\section{Etiología y Fisiopatología}

Su etiología aún no ha sido aclarada; Existe cada vez más evidencia de la participación de un factor genético. Se ha vinculado a diversos genes localizados en varios cromosomas. Se hace referencia a los marcadores D353050, localizados en el cromosoma 3p24.2-26 ${ }^{8}$, el D175939 del cromosoma 17q25, también en otras zonas del mismo cromosoma contenidas en la región 9-cM, de D 175785 a D1758369, y en el cromosoma 6 , en D6S44 $1^{10}$. Se propone una herencia poligénica vía autosómica dominante de baja penetrancia ${ }^{11}$. La presentación familiar, si bien ha sido notificada mayormente en población japonesa, también se han reportado casos en otras partes del mundo ${ }^{12}$. La descripción de un genotipo $\mathrm{G} / \mathrm{C}$ heterocigoto en la posición -418 en el promotor del inhibidor tisular de metaloproteinasa 2 (TIMP2) podría ser un factor genético predisponente para EM familiar ${ }^{13}$.

Diferentes estudios de tipo clínico, de laboratorio y anatomopatológicos han tratado de definir su patogénesis. Se ha descrito una íntima engrosada, en muestras de ACA de pacientes con moyamoya, con técnicas de inmunoreacción sugerentes de sobreexpresión de factor-1 (alfa), inductor de hipoxia, y endoglinas en la íntima de la $\mathrm{ACM}^{14}$. Junto con los factores genéticos mencionados, los factores ambientales también juegan su rol, como lo sugiere Ulrich, quien reporta que el 3,5\% de niños que habían recibido radioterapia por tumores cerebrales primarios, especialmente en las proximidades del polígono de Willis, desarrollaron EM en el seguimiento posterior, la cual se relacionó más con aquellos que tenían Neurofibromatosis tipo 1 y los que recibieron más de 5000 cGy de radiación ${ }^{15}$. Otros estudios se han dirigido a buscar moléculas solubles de adhesión en líquido cefalorraquídeo (LCR) de pacientes, lo que sugeriría un proceso inflamatorio en la etiología de esta enigmática enfermedad ${ }^{16}$, esto sería apoyado por la descripción de una EM por angiografía en una mujer de 20 años, 8 meses después de haber presentado una meningitis Neumocócica ${ }^{17}$. También se ha relacionado con apoptosis, evidenciada por la detección de caspasa-3 activada (por técnica de inmuno- rreacción) en la túnica media de la $\mathrm{ACM}$ con adelgazamiento de la pared vascular ${ }^{18} \mathrm{y}$ con la descripción de una alta proporción de anticuerpos anticélulas endoteliales en el suero de pacientes con $\mathrm{EM}^{19}$. Por otro lado, las publicaciones que reportan la persistencia de la arteria trigeminal ${ }^{20}$ y de la arteria Bernasconi-Cassinari, ambas arterias embrionarias, permite sospechar un inicio temprano de la enfermedad ${ }^{21}$.

\section{Clínica}

Aproximadamente el 60\% de los pacientes en edad pediátrica se presenta con crisis convulsivas focales, asociadas a crisis isquémicas transitorias o accidentes vasculares, las que serían precipitadas por hiperventilación en el llanto, desencadenándose vasoconstricción de los vasos sanguíneos del cerebro, los que por autorregulación se mantienen dilatados en forma permanente para compensar la isquemia crónica.

Otro síntoma de inicio, en un $6 \%$, es cefalea, probablemente secundaria a vasodilatación de vasos colaterales meníngeos que estimularían nociceptores de la duramadre. Un 2 a $4 \%$ debutan con hemorragia intracerebral ${ }^{1}$, a diferencia de los adultos cuya forma de presentación más frecuente es esta última ${ }^{2}$. En el momento de la presentación, la mayoría de los niños tienen compromiso bilateral en el estudio angiográfico, tal como se describe en nuestros casos, teniendo algunos el antecedente de un retraso en el desarrollo o deficiencias motoras focales sutiles ${ }^{7}$. Aunque generalmente el compromiso vascular es bilateral, se describen casos con afectación unilateral, tanto en la edad pediátrica como adulta, con progresión a enfermedad bilateral ${ }^{22}$. La incidencia de EM sintomática es desconocida, sin embargo, Satoshi Kuroda, condujo una investigación multicéntrica en Japón, en la cual se enrolaron 40 pacientes, 27 mujeres y 13 hombres (relación 2: 1 mujeres: hombres), quienes no habían tenido sintomatología cerebrovascular y su examen neurológico era normal. A estos pacientes que habían acudido al hospital por causas no relacionadas con EM, se les realizó una RNM y ARN. Treinta y siete pacientes tenían EM bilateral y 3 unilateral. Los casos que tenían cefalea, se estimó que 
era tensional en la mayoría, en contraste con el planteamiento de un cefalea vascular Se realizó bypass quirúrgico en 6 pacientes, los que no presentaron ningún episodio cerebrovascular en un período de seguimiento de 43,7 meses en promedio. De los 34 restantes, 7 tuvieron eventos cerebrovasculares ${ }^{3}$.

El pronóstico de este cuadro se relaciona en parte con la edad de comienzo de la sintomatología, a más temprana edad de inicio, peor pronóstico. Los embarazos y partos pueden empeorar el cuadro. Por otro lado, el tratamiento quirúrgico también interfiere en el pronóstico, retardando el avance de la enfermedad. Paralelamente, se describe la Vasculopatía moyamoya-like, o síndrome de Moyamoya, refiriéndose a una serie de condiciones, congénitas o adquiridas, asociadas a estenosis progresiva bilateral u oclusión de los segmentos terminales de la carótida interna, asociados a vasos colaterales anormales en la vecindad de la región ocluida. La historia natural y la patogénesis en estos casos, probablemente sea diferente a la de EM, dependiendo de la patología asociada. Tenemos como principales representantes de esta entidad clínica a el Síndrome de Down, (con una incidencia del 0,9-1,25\%, según 2 estudios japoneses), Neurofibromatosis tipo I, Esclerosis tuberosa, Síndrome de Marfan, Enfermedad de Hirschprung, Trombofilias, entre otras $^{23}$.

Este concepto clínico, nos obliga a investigar estas patologías frente a un niño en quien estamos planteando una EM.

\section{Estudio}

El estudio diagnóstico de la enfermedad de moyamoya, se realiza con angiografía convencional o angioresonanacia, las que muestran vasculopatía estenótica de las arterias carótidas internas, inmediatamente después de la salida de la arteria oftálmica ${ }^{24}$. En algunos casos también se ve comprometida la arteria basilar, El tejido cerebral se irriga por circulación colateral, proveniente mayormente de las arterias carótidas externas, arterias oftálmicas y arterias del territorio vertebrobasilar, aunque este esté comprometido ${ }^{25}$. La circulación colateral descrita, presenta una imagen similar al humo de cigarro, característica que explica el nombre de esta enfermedad ${ }^{4}$. Suziki y Takaka, dividieron las alteraciones angiográficas en 6 grados según la progresión de la oclusión carotídea ${ }^{26}$ : Estrechamiento de la bifurcación carotidea sin colaterales. Comienzo el fenómeno moyamoya con vasos colaterales de la base Intensificación del fenómeno moyamoya colaterales más prominentes, asociado a clínicamente, con la presentación sintomática de los pacientes. Reducción del fenómeno de moyamoya, todo el circulo de Willis y la arteria cerebral posterior, aparecen severamente estenóticas o completamente ocluidas, los vasos colaterales se estrechan, y aparece circulación colateral extracranial, siendo estas, la única fuente de irrigación cerebral.

Desaparición del fenómeno moyamoya, con oclusión completa de las carótidas cabe destacar que en las series publicadas, se ha visto la estabilidad de la sintomatología neurológica y de los cambios vasculares, no constatándose la desaparición del fenómeno de moyamoya ${ }^{27}$. El Electroencefalograma, tiene algunas características específicas de la edad pediátrica, a saber, enlentecimiento posterior o centrotemporal, patrón de ondas lentas monofásicas inducidas por la hiperventilación, y el fenómeno de rebote, después de la hiperventilación, caracterizado por la reaparición de ondas lentas, luego de la atenuación de este patrón, para volver a actividad basal luego de 10 minutos. Estos hallazgos electrofisiológicos, se encuentran en 50\% de los niños con $\mathrm{EM}^{28}$.

\section{Tratamiento}

La posibilidad de secuela neurológica permanente luego de un infarto cerebral extenso, es alta, por lo que debe procurarse evitarlos mediante tratamiento precoz, respecto del momento de diagnóstico, pese a que se describen pacientes con estabilización clínica una vez hecho el diagnóstico, pero esto en pacientes que han tenido un infarto considerable.

A pesar de que no existe tratamiento farmacológico para revertir o evitar la progresión del fenómeno moyamoya, se han usado anticoagulantes, antiagregantes plaquetarios y vasodila- 
tadores. El uso de aspirina se fundamenta en la presencia de émbolos trombóticos formados en los sitios estenóticos arteriales, y forma parte del esquema terapéutico aplicado en The Children's Hospital, Boston, ya sea como tratamiento de mantención, o como preparación prequirúrgica. En esta institución, también ha usado heparina de bajo peso molecular, en dosis de $0,5 \mathrm{mg} / \mathrm{kg}$ dos veces al día, subcutánea, en pacientes neurológicamente inestables, que requieren anticoagulación rápidamente reversible previo a la cirugía ${ }^{1}$. Como vasodilatadores, se han usado los bloqueadores de los canales de calcio, especialmente en aquellos pacientes que presentan cefalea vascular de difícil manejo, y como preventivo en caso de Accidentes Isquémicos Recurrentes ${ }^{29}$.

E1 tratamiento quirúrgico es recomendado especialmente en los pacientes con eventos isquémicos cerebrales, progresivos o repetidos, asociados con reducción de la reserva de perfusión del encéfalo. Las diferentes técnicas quirúrgicas ideadas, tiene como finalidad detener la injuria isquemica cerebral aumentando el flujo colateral a áreas hipoperfundidas de la corteza cerebral, usando la circulación de la carótida externa como donante. Se pueden clasificar en Directa e Indirectas ${ }^{1}$ :

a. Directas: La más común es la anastomosis de la Arteria Temporal Superficial a la ACM. Este procedimiento, si bien es cierto, es eficiente en proveer irrigación inmediata a las zonas con hipoperfusión, es técnicamente difícil, dado que las arterias donantes y receptoras, son de bajo calibre. Por otro lado, debido a la estenosis proximal, la nueva vía de irrigación provista por una rama sola de la ACM, no puede difundir a áreas más alejadas del cerebro. La oclusión temporal de la rama de ACM, durante el procedimiento, puede interferir con la vía colateral leptomeníngea presente, y llevar a un episodio isquémico perioperatorio.

b. Indirectas: Se describen varias alternativas: Encéfalo duro arteriosinangiosis: Se diseca la Arteria Temporal Superficial, y se sutura al borde del corte de la duramadre.

Encéfalomiosinangiosis: se diseca el músculo temporal ubicándolo en la superficie del cerebro, con el fin de inducir el desarrollo de vasos colaterales.
Encéfalomioarteriosinangiosis: Combinación de las anteriores.

Craneotomía con inversión de la dura: en los sitios donde se espera revascularización desde la dura al cerebro.

En el caso de la paciente $\mathrm{N}^{\circ} 1$, se efectuó una Encefaloduroarterio anastomosis derecha, y en un segundo acto quirúrgico a izquierda, no habiendo tenido posteriormente nuevos eventos vasculares en los 5 años de seguimiento.

$\mathrm{Al} 2^{\circ}$ paciente se aplicó la Trepanación de hemicalota bilateral simétrica (21 trepanaciones) con apertura de duramadre e inversión, también sin nuevos eventos vasculares hasta el momento de esta publicación.

\section{Comentario}

Nuestros pacientes debutaron con Crisis Convulsivas Focales y AVE isquémico. La primera paciente presentaba previamente cefalea mixta. El último paciente tenía un retraso psicomotor con compromiso motor y del lenguaje. Cabe hacer notar que a ambos se leshabía tomado una Tomografía cerebral sin contraste un año antes de su debut como parte del estudio de los síntomas descritos. Sin embargo, la Resonancia Magnética de cerebro, realizada en su primera hospitalización, presentaba imágenes de infartos antiguos bilaterales de territorio anterior. Podemos concluir entonces, que aunque la EM es infrecuente, es necesario tenerla presente cuando haya mínimos síntomas neurológicos, para los cuales no hay explicación. De acuerdo a lo analizado anteriormente, un diagnóstico precoz, un manejo clínico adecuado, un monitoreo eficiente y tratamiento quirúrgico antes de tener infarto o hemorragia cerebral, mejoran notoriamente el pronóstico, tanto en tiempo como en calidad de vida.

\section{Referencias}

1.- Smith ER MD, Scott RM MD: Surgical management of Moyamoya syndrome, Skull base and interdisciplinary approach 2005; 15 (1): 15-26.

2.- Yoshida $Y M D$, Yoshimoto $T M D$, Shirane $R M D$, Sakurai Y MD: Clinical course, Surgical management, and long term outcome of Moyamoya patients with 
rebleeding after an episode of intracerebral hemorrhage. Stroke 1999; 30: 2272-6.

3.- Satoshi Kuroda MD, Nobuo Hashimoto MD PhD, Takashi Yoshimoto MD PhD: Radiological Findings, Clinical Course, and Outcome in Asymptomatic Moyamoya Disease. Stroke 2007; 38: 1430-5.

4.- Susuki J, Takaku A: Cerebrovascular Moyamoya Disease. Disease showing abnormal net-like vassels in base of brain. Arch Neurol 1969; 20: 288-9.

5.- Nagaraja D, Verma A, Taly AB, Kumar $M V$, Jayakumar PN: Cerebrovascular disease in children. Acta Neurol Scand 1994; 90 (4): 251-5.

6.- Yonekawa Y, Ogata N, Kaku Y, Taub E, Imhof HG: Moyamoya disease in Europe, past and present status. Clin Neurol Neurosurg 1997; 99 Suppl 2: S58-60.

7.- Han DH, Know OK, Byun BJ, et al: A co-operative study; clinical characteristics of 334 Korean patients with moyamoya disease treated at neurosurgical institutes (1976-1994). The corean Society for Cerebrovascular disease. Acta neurochir 2000; 142: 1263-73.

8.- Ikeda H, Sasaki T, Yoshimoto, Fukui M, Arinami T: Mapping of familial moyamoya disease gene to chromosome 3p24.2-p26. Am J Hum Genet 1999; 64: 533-7.

9.- Yamauchi T, Tada M, Houkin K, et al: Linkage of familial moyamoya disease (spontaneus occlusion of the circles of Willi) to chromosome17q25. Stroke 2000; 31:930-5.

10.- Inoue TK, Ikesaki K, Sasazuki T, Matsushima T, Fukui M: Linkage analysis of moyamoya disease on chromosoma 6. J Child Neurol 2000; 15: 179-82.

11.- Osawa M, Kanai N, Kawai M, Fukuyama Y: Clinical genetic study on the idiopathic occlusion of the circle of the circles of Willis. The Researsh Committee of Spontaneus Occlusion of the circle of Willi (moyamoya disease) of the Ministry of Health and Welfare Japan. Annual, 1992; p 147-52.

12.- Pascual-Catroviejo I, Velázquez R: Síndrome de moyamoya. Seguimiento de 12 pacientes. Neurología 2006; 21 (10): 695-703.

13.- KanHyun-Seung K MD, Seung-Ki K MD, PhD, ByungKyu CH MD, PhD, Young-Yim K MS, Yong-Seung $H$ $M D, P h D$, Kyu-Chang $W M D, P h D$ : Single nucleotide polymorphisms of tissue inhibitor of metalloproteinase genes in familial moyamoya disease. Neurosurgery 2006; 58 (6): 1074-80.

14.- Yasushi T MD, PhD, Ken-ichiro K MD, PhD, Kazuhiko $N M D, P h D$, et al: Expression of hypoxia-inducing factor-1[alpha] and endoglin in intimal hyperplasia of the middle cerebral artery of patients with moyamoya disease.[Miscellaneous Article]. Neurosurgery 2007; 60 (2): 338-45.

15.- Ullrich NJ MD, PhD, Robertson R MD, Kinnamon $D D M S$, et al: Moyamoya following cranial irradiation for primary brain tumors in children. [Article] Neurology 2007; 68(12): 932-8.

16.- Kouichi O MD, Shinji N MD, Rieko A PhD, Naozumi I DDS, PhD, Masaru K MD, Yoshio H DDS, PhD: Anti-[alpha]-Fodrin Autoantibodies in Moyamoya Disease. [Report]. Stroke 2003; 34 (12): e244-e246.

17.- Postinfectious vasculopathy with evolution to moyamoya syndrome. [Report] Journal of Neurology, Neurosurgery \& Psychiatry 2005; 76(2): 256-9.

18.- Yasushi T MD, PhD, Ken-ichiro K MD, PhD, Nobutake $S M D, P h D$, Kazuhiko $N$ MD, PhD, Nobuo $H M D$, $P h D$ : Caspase-3-dependent Apoptosis in Middle Cerebral Arteries in Patients with Moyamoya Disease. [Miscellaneous Article] Neurosurgery 2006; 59 (4): 894-901.

19.- Soriano $S G M D$, Cowan $D B P h D$, Proctor $M R M D$, Scott RM MD: Levels of Soluble Adhesion Molecules Are Elevated in the Cerebrospinal Fluid of Children with Moyamoya Syndrome. Neurosurgery 2002; 50 (3): 544-9.

20.- Kwak R, Kadoya S: Moyamoya disease associated with persistent primitive trigeminal artery. Report of two cases J Neurosurg 1983; 59: 166-71.

21.- Pascual-Castroviejo I, Pascual-Pascual SI, Pérez Higueras A, Martinez $V$ : Moyamoya disease with a marker collateral supply thought the artery of BernasconiCassinari. Brain Dev 1966; 18: 71-4.

22.- Kelly ME, Teresa E: Bell-Stephens Progresion of unilateral Moyamoya Disease: A Clinical Series, Cerebrovascular Dis 2006; 22: 109-15.

23.- Horn P, Pfister S, Bueltmann E, Vajkoczy $P$, Schmiedek $P$ : Moyamoya-like vasculopathy (moyamoya syndrome) in children. Childs Nerv Syst 2004; 20: 382-91.

24.- Pascual-Castroviejo I, López Martín V, Rodríguez Costa T, Tendero A, Palencia R: Síndrome de Nishmoto moyamoya. A propósito de un caso con estudio angiográfico completo. Radiología 1973; 15: 35-42.

25.- Miyamoto S, Kiruchi H, Karasawa J, Nagata I, Ikota T: Study of the posterior circulation in moyamoya disease. Clinical and neuroradiological evaluation. J Mneurosurg 1984; 61: 1032-7.

26.- Suzuki J, Takaku A: Cerbovascular Moyamoya Disease. Disease showing abnormal net like vassels in base of brain. Arch Neurol 1969; 20: 288-99.

27.- Ezura M, Yoshimoto T, Fujiwara S, Takahashi A, Shirane R, Mizoi K: Clinical and Angiographic followup of childhood onset moyamoya disease, Child's nerv Syst 1995; 11: 591-4.

28.- Kodoma N, Aoki Y, Hiraga H, Wada T, Suzuki J: Electroencephalographic Findings in Children with Moyamoya Disease. Arch Neurol 1979; 36: 16-9.

29.- Scott RM: Moyamoya Syndrome, a surgically treatable cause of stroke in pediatric patient. Clin Neurosurg 2000; 47: 378-84. 